\title{
Mikrodalga Radyasyonun Un biti, Tribolium castaneum (Herbst, 1797) (Coleoptera: Tenebrionidae)'a Etkisi
}

\section{Effect of Microwave Radiation to Red Flour Beetle Tribolium castaneum (Herbst) (Coleoptera: Tenebrionidae)}

Geliş / Received: 28/01/2021

\author{
Emre Karadağ $\breve{1}^{1}$, Ali Kayahan ${ }^{2 *}$
}

Revize / Revised: 02/06/2021

Kabul / Accepted: 03/06/2021

$\ddot{\text { ÖZ }}$

Depolanmış ürünlerin korunması oldukça önemlidir. Bu ürünler üzerinde kayıplara sebep olan zararlılara karşı mücadelede üreticiler pestisitleri tercih etmektedir. Ancak araştırmalar bu kimyasalların çevre ve insan sağlı̆̆ açısından olumsuz etkilerini ortaya koymaktadır. Bunun önüne geçebilmek için son yıllarda araştırıcılar alternatif yöntemlere yönelmektedir. $\mathrm{Bu}$ yöntemlerden biri olan ışınlanma teknikleri, zararlılar üzerinde kısırlaştırıcı, gelişimi engelleyici ve öldürücü etkiye sahiptir. Bununla birlikte zararlılarda direnç ve ürünler üzerinde kalıntı oluşturmama gibi avantajları vardır.

Bu çalışmada mikrodalga ışınlanmasının Tribolium castaneum (Herbst, 1797) (Coleoptera: Tenebirionidae) üzerindeki etkisini ortaya koymak amacıyla ergin bireyler değişik güç ve sürelerde radyasyona maruz bırakılmıştır. Denemelerde mikrodalga radyasyonu için 5 değer $(120,350,460,600,700$ watt), süre olarak da 7 süre $(5,10,20$, 40, 60, 80, $100 \mathrm{sn}$ ) belirlenmiş ve uygulanmıştır. Denemeler kurulduktan bir saat sonra canlı ve ölü bireyler sayılıp kaydedilmiştir. Elde edilen verilere bakıldığında radyasyon miktarı ve uygulama süresi arttıkça meydana gelen ölümlerin de arttı̆̆ belirlenmiştir.

$\mathrm{Bu}$ çalışma sonucunda elde edilen veriler incelendiğinde fiziksel bir mücadele yöntemi olan mikrodalga radyasyonunun, T. castaneum ergin bireyleri üzerinde öldürücü etki gösterdiği saptanmış ve yoğun şekilde uygulanan kimyasal mücadeleye alternatif bir yöntem olarak kullanılabileceği kanısına varılmıştır.

Anahtar Kelimeler- Depo Zararlıları, Fiziksel Mücadele, Mikrodalga Radyasyonu, Tribolium Castaneum

\begin{abstract}
Preservation of stored products is very important. Manufacturers prefer pesticides against pests that cause losses on these products. However, recent researches have revealed the negative effects of these chemicals in terms of environment and human health. Researchers have been turned to alternative methods in recent years to prevent this. Radiation techniques, one of these methods, have a sterilizing, growth-inhibiting, and lethal effect on the pests. Besides, these techniques have advantages, such as not causing resistance to pests and no residue on products.
\end{abstract}

In this study, adult individuals were exposed to radiation at different power and time with the intent to reveal the effect of microwave irradiation on Tribolium castaneum (Herbst, 1797) (Coleoptera: Tenebirionidae). In the trials,

1İletişim: emrekaradag75@ hotmail.com (https://orcid.org/0000-0002-9786-1143)

Bitki Koruma Bölümü, Yozgat Bozok Üniversitesi, Ziraat Fakültesi, Yozgat, Türkiye

2*Sorumlu yazar iletişim: aalikayahan@gmail.com (https://orcid.org/0000-0002-3671-254X)

Bitki Koruma Bölümü, Yozgat Bozok Üniversitesi, Ziraat Fakültesi, Yozgat, Türkiye 
5 values $(120,350,460,600,700$ watts) and 7 times $(5,10,20,40,60,80,100$ seconds) for microwave radiation were determined and applied. One hour after the trials were set up, live and dead individuals were counted and recorded. Considering the data obtained, it was determined that the amount of radiation and the duration of the application increased, and the deaths that occurred increased.

When the data obtained as a result of this study are examined, it is determined that microwave radiation, which is a physical control method, has a lethal effect on adult individuals of T. castaneum, and can be used as an alternative method to the intensively used chemical control.

\section{Keywords- Storage Pests, Physical Control, Microwave Radiation, Tribolium castaneum}

\section{GİRiş}

Tarımsal üretim içerisinde tahıl üretimi hem dünya genelinde hem de ülkemizde ilk sıralarda yer almaktadır. Ülkemizde üretimi ilk sıralarda yer alan tahılların başında arpa ve buğday gelmektedir. Yaklaşık 70 milyon dekar alanda tarımı yapılan buğdayın yıllık üretimi 20 milyon 500 bin tondur. İkinci ürün olan arpanın ise yaklaşık 31 milyon dekar alanda tarımı yapılmakta olup, yıllık 8 milyon 300 bin ton üretilmektedir [1].

Dünya genelinde insan nüfusu gün geçtikçe artış göstermekte ve buna bağlı olarak ihtiyaç duyulan besin miktarı da artmaktadır. Bu sebeple tarımsal üretim yapılan alanlardan yüksek oranda verim elde edebilmek için tarımsal ürünlerin zararlılardan korunması önemlidir [2]. Zararlılarla mücadelede kimyasalların yoğun bir şekilde kullanılması insan ve çevre sağlığı açısından olumsuz koşullar meydana getirmektedir [3-6].

Artan üretimle birlikte tahılların hasat sonrası depolanması sırasında ürünlerdeki kalite ve verim kayıplarının oluşmasında böcekler ve akarlar önemli etmenler arasındadır. Bahsedilen bu zararlıların ürünlerde yıllık ortalama \%10 kayba neden oldukları kabul edilmektedir [7]. Tahılların hasattan tüketilinceye kadar geçen sürede en az kayıp verdirilerek depolanması ve korunması oldukça önemlidir [8]. Depolanmış ürünler üzerinde beslenen zararlılar doğrudan veya dolaylı şekilde zarar meydana getirmektedir. Üründe tohumluk özelliğin düşmesine, ağırlık kayıplarına, kalite ve besin değerlerinde olumsuz yönde değişimlere yol açarak ticari değerin düşmesine sebep olmaktadırlar [9]. Bu zararlılarla mücadelede üreticiler çoğunlukla kimyasal uygulamalardan olan fumigasyon yöntemi kullanılarak mücadele etmeyi tercih etmektedir [11]. Yoğun şekilde insektisit kullanımı çevre ve insan sağlığının olumsuz etkilemenin yanında hem ürünler üzerinde kalıntı problemlerine hem de zararlıların direnç kazanmalarına neden olmaktadır [10, 12-15].

Tarımsal zararlı organizmalara karşı uygulanan radyasyon tekniği kimyasal mücadeleye alternatif savaşım yöntemleri arasındadır ve literatürde zararlılar üzerinde yapılmış çalışmalar göze çarpmaktadır [16-22]. Bu teknik zararlıya karşı uygulandığında böcekler üzerinde farklı etkiler (kısırlaştırma, gelişimi engelleme ve öldürme) göstermektedir [23, 24]. Kullanılan bu yöntemde uygulanan 1şın zararlıya iyi bir şekilde nüfuz etmekte ve hem ürünün içindeki hem de dişındaki böcekleri öldürebilmektedir [25]. Bu çalışmanın da ana materyali olan Tribolium castaneum (Herbst) ve farklı depo zararlıları üzerinde mikrodalga radyasyonun etkileri ile ilgili bazı çalışmalar yürütülmüş ve olumlu sonuçlar elde edilmiştir [26-36].

Un biti T. castaneum, depolanmış ürünlerde özellikle de buğdaydan elde edilen ürünler (un, makarna) üzerinde zarara neden olmaktadır. Bu tür aynı zamanda kurutulmuş meyve, bisküvi ve fındık gibi ürünlerde de zarar yapmaktadır [12, 37, 38]. Ayrıca bu türün erginleri koku bezlerinden kötü kokulu bir sıvı salgılayarak üründe kokuşmaya ve küflenmeye neden olmaktadır [34].

$\mathrm{Bu}$ çalışma, mikrodalga radyasyonunun, önemli bir zararlı olan $T$. castaneum üzerindeki öldürücü etkisinin araştırılması ve zararlının kimyasal mücadeleye alternatif bir yöntemle kontrol edilmesine katkı sağlamak amacıyla planlanmıştır.

\section{MATERYAL VE YÖNTEM}

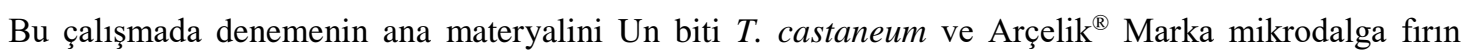
oluşturmaktadır. 


\section{A. Tribolium Castaneum Üretimi}

Tribolium castaneum ergin bireyleri Yozgat Bozok Üniversitesi, Ziraat Fakültesi, Bitki Koruma Bölümü'nde üretilmekte olan stok kültürden sağlanmıştır. Kitle üretimden alınan bireyler (Erkek-Dişi), içerisinde 1:1:1 oranında bisküvi:kepek:un karışımı bulunan $10 \times 15 \times 10 \mathrm{~cm}$ ölçülerindeki plastik kaplara alınmış ve yumurta bırakmaları beklenmiş̧tir. Bırakılan yumurtadan çıkan larvaların aynı ortamda ergin olmaları sağlanarak kültür oluşturulmuştur. Denemelerde kullanılan ergin bireyler bu kültürden temin edilmiştir. Üretimlerin tamamı $25 \pm 1{ }^{\circ} \mathrm{C}, \% 60 \pm 5$ orantılı nem 16:8 (aydınlık:karanlık) aydınlatma koşullarına sahip odada gerçekleştirilmiştir.

\section{B. Denemelerin Kurulmast}

Tribolium castaneum'un kitle üretiminden elde edilen ergin bireyler denemede kullanılmak üzere ayrı ayrı petri kaplarına alınmıştır. Petri kaplarının üst kısımlarına uygulanacak olan mikrodalga radyasyonunun gücü ve tekerrür sayısı asetat kalemiyle yazılmıştır. Daha sonra firın uygulanacak güce ayarlanmış ve içerisinde böcek olan petri kapları belirlenen süre içerisinde mikrodalga firın içerisinde tutulmuştur. Denemelerde 5 farklı güç (120, $350,460,600,700$ watt) ve 7 farklı süre $(5,10,20,40,60,80,100 \mathrm{sn})$ belirlenmiş ve uygulanmıştır (Radyasyon değerleri belirlenirken cihaz üzerindeki değerler esas alınmıştır). Kontrol uygulamasında besin ile birlikte ayrı petri kaplarına alınan bireylerin durumları gözlenmiştir. Denemeler her bir uygulama için 10 tekerrür olacak şekilde düzenlenmiştir. Radyasyon uygulandıktan bir saat sonra petriler içerisindeki canlı ve ölü bireyler sayılıp kaydedilmiştir.

\section{C. İstatistiksel Analizler}

Denemelerde her bir uygulama ve kontrol için ayrı ayrı tablo hazırlanarak süre ve radyasyon güçlerine göre canlı ve ölü bireyler kaydedilmiştir. Kaydedilen veriler ile uygulanan radyasyon güçlerinin farklı sürelerdeki etkileri kontrol ile birlikte karşılaştııılmış ve her bir radyasyon gücünde süreler arasındaki etkinlik farkları belirlenmiştir. Buna ek olarak farklı radyasyon güçlerinin aynı süre içerisindeki etkileri de analiz edilerek aynı sürede farklı güçlerin etkileri saptanmıştır. Elde edilen verilerin karşılaştırılmasında tek yönlü varyans analizi ANOVA uygulanmış ve farkın önemli bulunması durumunda Tukey çoklu karşılaştırma testi ile gruplandırma yapılmıştır. Elde edilen verilere bakılarak uygulaması yapılan mikrodalga radyasyon değerleri ve sürelerinin $T$. castaneum üzerindeki etkileri belirlenmiştir. İstatistiksel analizlerin yapılmasında Minitab (Ver. 17) programından yararlanılmıştır [39].

\section{BULGULAR VE TARTIŞMA}

Bu çalışmada uygulama yapıldıktan sonra canlı ve ölü bireyler sayılmış ve kaydedilmiştir. Çalışma sonucunda elde edilen verilere göre uygulaması yapılan bütün mikrodalga seviyelerinin uygulama süreleri arasında istatistiksel bir fark olduğu saptanmıştır $(p<0.05)$. Elde edilen verilere göre mikrodalga düzeylerinin uygulama süreleri arttıkça ortaya çıkan ölüm oranının da arttığı gözlenmiştir. Sonuçlara göre 120 watt değerinde 40-100 sn aralığında ölüm oranları benzerken; bu değerlerin 5-10 sn aralığındaki ölüm oranlarından daha yüksek olduğu gözlenmiştir. 350 ve 460 watt değerlerindeki ölüm oranları son iki sürede $(80,100 \mathrm{sn})$ benzerken; elde edilen bu değerlerin diğer uygulama sürelerinden farklı olduğu belirlenmiştir. 600 ve 700 watt değerlerinde $T$. castaneum bireylerinin tamamının 40 sn'den sonraki sürelerde öldüğü gözlenirken; ilk üç uygulama süresinde $(5,10,20$ sn) ölüm oranının diğer sürelerden düşük ve farklı olduğu görülmüştür (Tablo 1).

Analizlerin ikinci kısmında aynı uygulama süresi içinde farklı radyasyon değerlerinin karşılaştırılması yapılmıştır. Buna göre sürelere bağlı olarak radyasyon gücü arttıkça ölümlerin de arttığı gözlenmiştir $(p<0.05)$. Buna göre radyasyon 5 sn uygulandığında 120 watt değerindeki ölü birey sayısı diğger radyasyon değerlerine göre farklı ve en düşük sayıda olmuştur. Bu uygulama süresi içinde radyasyon değeri arttıkça ölüm miktarı da artış göstermiş̧tir. Uygulama süresi 10 sn olduğunda 700 watt değerindeki ölü birey sayısı diğerlerine göre farklı ve en yüksek sayıda olmuştur. 40-100 sn aralığındaki uygulama sürelerinde özellikle yüksek radyasyon değerlerinde ölümlerin çok yüksek olduğu hatta bazı değerlerden uygulama yapılan bireylerin tamamının öldüğü görülmektedir (Tablo 2). 
Tablo 1. Uygulamalar sonrasında yapılan sayımlara göre ortalama ölü birey sayıları (Sürelere göre). (Ort $\pm \mathrm{SH})$

\begin{tabular}{|c|c|c|c|c|c|c|c|c|}
\hline \multicolumn{9}{|c|}{ Süreler } \\
\hline & $5 \mathrm{sn}$ & $10 \mathrm{sn}$ & $20 \mathrm{sn}$ & $40 \mathrm{sn}$ & $60 \mathrm{sn}$ & $80 \mathrm{sn}$ & $100 \mathrm{sn}$ & Kontrol \\
\hline 120 & $3.2 \pm 0.51$ & $5.2 \pm 0.47$ & $7.1 \pm 0.23$ & $7.3 \pm 0.37$ & $7.6 \pm 0.16$ & $8.0 \pm 0.92$ & $9.0 \pm 0.47$ & $0.00 \pm 0.00$ \\
\hline Watt & $\mathrm{c}$ & bc & $a b$ & $\mathrm{a}$ & a & $\mathrm{a}$ & $\mathrm{a}$ & d \\
\hline 350 & $3.6 \pm 0.34$ & $6.4 \pm 0.22$ & $7.6 \pm 0.27$ & $8.3 \pm 0.21$ & $9.1 \pm 0.28$ & $9.9 \pm 0.10$ & $10.0 \pm 0.00$ & $0.00 \pm 0.00$ \\
\hline Watt & $\mathrm{e}$ & d & $\mathrm{c}$ & $\mathrm{bc}$ & $a b$ & $\mathrm{a}$ & $\mathrm{a}$ & f \\
\hline 460 & $4.7 \pm 0.34$ & $6.7 \pm 0.21$ & $8.1 \pm 0.18$ & $9.1 \pm 0.28$ & $9.6 \pm 0.22$ & $10.0 \pm 0.00$ & $10.0 \pm 0.00$ & $0.00 \pm 0.00$ \\
\hline Watt & $\mathrm{e}$ & d & $\mathrm{c}$ & $\mathrm{b}$ & $a b$ & $\mathrm{a}$ & $\mathrm{a}$ & f \\
\hline 600 & $6.6 \pm 0.27$ & $7.4 \pm 0.27$ & $8.4 \pm 0.31$ & $9.8 \pm 0.13$ & $10.0 \pm 0.00$ & $10.0 \pm 0.00$ & $10.0 \pm 0.00$ & $0.0 \pm 0.00$ \\
\hline Watt & d & $\mathrm{c}$ & $\mathrm{b}$ & a & a & $\mathrm{a}$ & $\mathrm{a}$ & $\mathrm{e}$ \\
\hline 700 & $7.1 \pm 0.23$ & $8.5 \pm 0.17$ & $9.1 \pm 0.27$ & $10.0 \pm 0.00$ & $10.0 \pm 0.00$ & $10.0 \pm 0.00$ & $10.0 \pm 0.00$ & $0.00 \pm 0.00$ \\
\hline Watt & c & b & b & $\mathrm{a}$ & $\mathrm{a}$ & $\mathrm{a}$ & $\mathrm{a}$ & d \\
\hline
\end{tabular}

Aynı satırdaki farklı harfler istatistiksel olarak bir farkın olduğunu göstermektedir. $\left(\mathrm{F}_{120 \text { watt: }}: 40.79 ; \mathrm{P}_{120 \text { watt }}: 0.001 ; \mathrm{F}_{350 \text { watt }}: 263.01 ; \mathrm{P}_{350 \text { watt }}: 0.001 ;\right.$ $\left.\mathrm{F}_{460 \text { watt }}: 304.79 ; \mathrm{P}_{460 \text { watt }}: 0.001 ; \mathrm{F}_{600 \text { watt }}: 366.83 ; \mathrm{P}_{600 \text { watt }}: 0.001 ; \mathrm{F}_{7000 \text { watt }}: 591.31 ; \mathrm{P}_{700 \text { watt }}: 0.001\right)$ (Ort: Ortalama, SH: Standart hata, sn: saniye)

Tablo 2. Uygulamalar sonrasında yapılan sayımlara göre ortalama ölü birey sayıları (Radyasyon değerlerine göre). (Ort $\pm \mathrm{SH})$

\begin{tabular}{|c|c|c|c|c|c|c|c|}
\hline & $5 \mathrm{sn}$ & $10 \mathrm{sn}$ & $20 \mathrm{sn}$ & $40 \mathrm{sn}$ & $60 \mathrm{sn}$ & $80 \mathrm{sn}$ & $100 \mathrm{sn}$ \\
\hline 120 Watt & $3.2 \pm 0.51$ & $5.2 \pm 0.47$ & $7.1 \pm 0.23$ & $7.3 \pm 0.37$ & $7.6 \pm 0.16$ & $8.0 \pm 0.92$ & $9.0 \pm 0.47$ \\
\hline $120 \mathrm{Watt}$ & $\mathrm{c}$ & $\mathrm{c}$ & $\mathrm{c}$ & $\mathrm{c}$ & $\mathrm{c}$ & $\mathrm{b}$ & b \\
\hline 350 Watt & $\begin{array}{c}3.6 \pm 0.34 \\
\text { bc }\end{array}$ & $\begin{array}{c}6.4 \pm 0.22 \\
b\end{array}$ & $\begin{array}{c}7.6 \pm 0.27 \\
b c\end{array}$ & $\begin{array}{c}8.3 \pm 0.21 \\
b\end{array}$ & $\begin{array}{c}9.1 \pm 0.28 \\
b\end{array}$ & $\begin{array}{c}9.9 \pm 0.10 \\
\mathrm{a}\end{array}$ & $\begin{array}{c}10.0 \pm 0.00 \\
\mathrm{a}\end{array}$ \\
\hline 460 Watt & $\begin{array}{c}4.7 \pm 0.34 \\
\mathrm{~b}\end{array}$ & $\begin{array}{c}6.7 \pm 0.21 \\
\mathrm{~b}\end{array}$ & $\begin{array}{c}8.1 \pm 0.18 \\
\text { abc }\end{array}$ & $\begin{array}{c}9.1 \pm 0.28 \\
a b\end{array}$ & $\begin{array}{c}9.6 \pm 0.22 \\
a b\end{array}$ & $\begin{array}{c}10.0 \pm 0.00 \\
\mathrm{a}\end{array}$ & $\begin{array}{c}10.0 \pm 0.00 \\
\mathrm{a}\end{array}$ \\
\hline 600 Watt & $\begin{array}{c}6.6 \pm 0.27 \\
\mathrm{a}\end{array}$ & $\begin{array}{c}7.4 \pm 0.27 \\
\mathrm{ab}\end{array}$ & $\begin{array}{c}8.4 \pm 0.31 \\
a b\end{array}$ & $\begin{array}{c}9.8 \pm 0.13 \\
\mathrm{a}\end{array}$ & $\begin{array}{c}10.0 \pm 0.00 \\
\mathrm{a}\end{array}$ & $\begin{array}{c}10.0 \pm 0.00 \\
\mathrm{a}\end{array}$ & $\begin{array}{c}10.0 \pm 0.00 \\
\mathrm{a}\end{array}$ \\
\hline 700 Watt & $\begin{array}{c}7.1 \pm 0.23 \\
\mathrm{a}\end{array}$ & $\begin{array}{c}8.5 \pm 0.17 \\
\mathrm{a}\end{array}$ & $\begin{array}{c}9.1 \pm 0.27 \\
\mathrm{a}\end{array}$ & $\begin{array}{c}10.0 \pm 0.00 \\
\mathrm{a}\end{array}$ & $\begin{array}{c}10.0 \pm 0.00 \\
\mathrm{a}\end{array}$ & $\begin{array}{c}10.0 \pm 0.00 \\
\mathrm{a}\end{array}$ & $\begin{array}{c}10.0 \pm 0.00 \\
\mathrm{a}\end{array}$ \\
\hline
\end{tabular}

Aynı sütundaki farklı harfler istatistiksel olarak bir farkın olduğunu göstermektedir. $\left(\mathrm{F}_{5 \mathrm{sn}}: 24.88 ; \mathrm{P}_{5 \mathrm{sn}}: 0.001 ; \mathrm{F}_{10 \mathrm{sn}}: 18.16 ; \mathrm{P}_{10 \mathrm{sn}}: 0.001 ; \mathrm{F}_{20 \mathrm{sn}}: 8.89 ;\right.$ $\left.\mathrm{P}_{20 \mathrm{sn}}: 0.001 ; \mathrm{F}_{40 \mathrm{sn}}: 22.68 ; \mathrm{P}_{40 \mathrm{sn}}: 0.001 ; \mathrm{F}_{60 \mathrm{sn}}: 32.78 ; \mathrm{P}_{60 \mathrm{sn}}: 0.001 ; \mathrm{F}_{80 \mathrm{sn}}: 4.58 ; \mathrm{P}_{80 \mathrm{sn}}: 0.001 ; \mathrm{F}_{100 \mathrm{sn}}: 4.50 ; \mathrm{P}_{100 \mathrm{sn}}: 0.001\right)$ (Ort: Ortalama, SH: Standart hata, sn: saniye)

Bu çalışmada $T$. castaneum ergin bireyleri üzerinde farklı güç ve süreler içerisinde mikrodalga radyasyonun etkileri belirlenmiştir. Elde edilen veriler incelendiğinde radyasyon gücü ve süre arttıkça ölümlerin de arttığı saptanmıştır. Farklı depo zararlıları (T. castaneum, Cryptolestes ferrugineus, Sitophilus granarius) üzerinde mikrodalga radyasyonun etkisinin incelendiği bir çalışmada zararlılar farklı sürelerde farklı radyasyon derecelerine maruz bırakılmıştır. Uygulaması yapılan radyasyon değerlerinin $(250,300,400,500 \mathrm{w}) T$. castaneum üzerindeki etkileri incelendiğinde 400 ve 500 w değerlerinin uzun sürede daha öldürücü olduğu gözlenirken; 250 ve $300 \mathrm{w}$ değerlerinde uzun uygulama süresinde ölüm oranı \%77-90 arasında değişiklik göstermiştir [31]. Mikrodalga radyasyonunun $T$. castaneum üzerinde etkisinin incelendiği bir başka çalışmada, bu zararlının erginleri değişen güç ve sürelerde radyasyona maruz bırakılmıştır. Elde edilen sonuçlara göre mikrodalga radyasyonun gücü ve uygulama süresinin artması erginlerdeki ölüm oranını da artırmıştır. Çalışma sonunda çıkan verilere göre 400 w'lık uygulamada 56 sn sonunda erginlerin \%90'ının öldüğü gözlenmiştir [32]. Farklı mikrodalga radyasyon değerlerinin $T$. castaneum üzerindeki etkilerinin belirlenmeye çalışıldığı bir çalışmada, 150 w'lık radyasyona 180 sn maruz bırakılan erginlerin \%33'ü ölürken; aynı sürede 360 w’lık radyasyona maruz kalan erginlerin tamamı ölmüştür. 900 w’lık mikrodalga radyasyonuna 10 sn maruz bırakılan erginlerde ölüm oranı \%13.40 iken; bu oran 30 sn sonunda \%100 olmuştur. Çalışmanın sonucunda elde edilen verilere göre un biti ile bulaşık ürünlerin mikrodalga radyasyonu ile kısa sürede bu zararlıdan temizlenebileceği anlaşılmaktadır [26]. Farklı depo zararlılarından olan T. castaneum ve Rhizopertha dominica türleri üzerinde yapılan bir çalışmada, farklı sürelerde (2, 4, 6 ve $8 \mathrm{dk}) 200 \mathrm{w}$ radyasyon uygulanmış ve bireylerin ölüm oranları elde edilmiştir. Sürelere bağlı olarak $T$. castaneum için bu oranların sürelere bağlı olarak sırasıyla \%28.33, \%55, \%83.33 ve \%98.33 olduğu belirlenmiştir [35]. İki farklı depo zararlısı (T. castaneum, Oryzaephilus surinamensis) üzerinde yapılan bir çalışmada farklı radyasyon değerlerinin $(180,300,600,800 \mathrm{w})$ zararlılar üzerindeki letal etkileri belirlenmiştir. Elde edilen verilere göre üzerinde uygulama yapılan T. castaneum ergin bireylerinde ölüm oranının düşük olduğu gözlenirken; yüksek radyasyon seviyelerinde $(600,800 \mathrm{w})$ özellikle 40 s süreyle maruz bırakıldığında ölüm miktarının $\% 97$ ve $\% 100$ olduğu belirlenmiştir [40]. Tribolium castaneum, Sitophilus granaries ve Callosobruchus maculatus ile yapılan bir çalışmada farklı süre $(10,20,30,40,50$ ve 60 s) ve farklı radyasyon değerlerinin $(0,20,40,60,80 \mathrm{ve} 100 \mathrm{w})$ 
bu zararlılar üzerindeki etkileri incelenmiştir. Elde edilen verilere göre 30 sn uygulama süreleri ile $40 \mathrm{w}$ ve üzeri radyasyon değerlerinde $T$. castaneum için ölüm oranlarının \%90’ın üzerinde olduğu gözlenmiştir. Ölüm oranlarının özellikle yüksek uygulama süresi ve yüksek radyasyon dozlarında \%100 olduğu görülmektedir [41]. Mikrodalga radyasyonun etkilerinin incelendiği bir çalışmada farklı sürelerde uygulanan (10, 30 ve $50 \mathrm{sn})$ farklı radyasyon değerlerinin $(280,560$ ve $840 \mathrm{w})$ T. castaneum ve Trogoderma granarium üzerindeki letal etkileri belirlenmiştir. Elde edilen verilere göre her iki zararlı için en etkili radyasyon değerinin $840 \mathrm{w}$ olduğu tespit edilmiştir. 50 sn boyunca bu seviyeye maruz bırakılan T. castaneum için ölüm oranının \%90 olduğu saptanmıştır [42]. Yakın zamanda yapılan bir çalışmada farklı sürelerde (30-90 sn) uygulanan farklı radyasyon seviyelerinin (120-600 w) T. castaneum üzerindeki letal etkileri belirlenmiştir. Elde edilen verilere göre 60 ve 90 sn süreyle uygulanan 480 ve $600 \mathrm{w}$ radyasyon değerlerinin uygulamadaki bütün bireyleri öldürdüğü belirlenmiştir [36]. Bu çalışma sonucunda elde edilen verilerin tamamı incelendiğinde literatürde olduğu gibi fiziksel bir mücadele yöntemi olan mikrodalga radyasyonunun $T$. castaneum ergin bireyleri üzerinde öldürücü etki gösterdiği saptanmıştır. Bu çalışmada da literatürde olduğu gibi zararlının ergin bireyleri yüksek radyasyon değerlerinde uzun süre bırakıldığında yüksek ölüm oranları ortaya çıkmaktadır. Elde edilen verilerin un bitinin mücadelesinde kullanılan ve kimyasal savaşıma alternatif bir yöntem olan, radyasyonla mücadele yöntemine katkı sağlayacağı düşünülmektedir. Buna ek olarak özellikle ihracatta önemli bir kalem olan tahıllar üzerinde kalıntı sorunun çözümü için yardımcı veriler oluşturulması açısından faydalı olacağı kanaatine varılmıştır.

\section{KAYNAKLAR}

[1] Türkiye İstatistik Kurumu. (2020). Tahıllar ve Diğer Bitkisel Ürünlerin Alan ve Üretim Miktarları. https://biruni.tuik.gov.tr/medas/?kn=92\&locale=tr.

[2] Schöller, M., Prozell, S., Al-Kirshi, A. G. \& Reichmuth, C. (1997). Towards biological control as a major component of integrated pest management in stored product protection. Journal of Stored Products Research, 33(1), 81-97.

[3] Moore, D. \& Prior C. (1993). The potential of mycoinsecticides. Biocontrol News and Information, 14, 3140.

[4] Arthur, F. H. (1996). Grain protectants: current status and prospects for the future. Journal of Stored Products Research, 32, 293-302.

[5] Zettler, J. L. \& Arthur, F. H. (2000). Chemical control of stored product insects with fumigants and residual treatments. Crop Protection, 19, 577-582.

[6] Ayvaz, A., Albayrak, S. \& Karabörklü, S. (2008). Gamma radiation sensitivity of the eggs, larvae and pupae of Indian meal moth Plodia interpunctella (Hübner) (Lepidoptera: Pyralidae). Pest Management Science, 64, 505-512.

[7] Donahaye, E. J. \& Messer, E. (1992). Reduction in grain storage losses of small-scale farmers in tropical countries. Research Report RR-91-7, The Allan Shawn Feinstein World hunger Program, Brown University.

[8] Bağc1, F., Yılmaz, A. \& Ertürk, S. (2014). Ankara ili hububat depolarında bulunan zararlı böcek türleri. Bitki Koruma Bülteni, 54(1), 69-78.

[9] Boxall, R. A. (2001). Post-harvest losses to insect-a world overview. International Biodeterioration \& Biodegradation, 48, 137-152.

[10] Emekçi, M. \& Ferizli, A. G. (2000). Current status of stored products protection in Turkey. IOBC wprs Bulletin, 23(10), 39-46.

[11] Banks, H. J. (1994). Fumigation-an endangered technology. Proceedings of the 6th International Working Conference on Stored-Product Protection, 17-23 April 1994, Canberra, Australia.

[12] Sinha, R. N. \& Watters, F. L. (1985). Insect Pests of Flour Mills, Grain Elevators, and Feed Mills and Their Control. Agriculture Canada, Winnipeg, M.B., Canada.

[13] Hansen, L. S. \& Jensen, K. M. V. (2002). Effect of Temperature on Parasitism and Host-Feeding of Trichogramma turkestanica (Hymenoptera: Trichogrammatidae) on Ephestia kuehniella (Lepidoptera: Pyralidae). Journal of Economical Entomology, 95(1), 50-56.

[14] Fields, P. G. \& White, N. D. G. (2002). Alternatives to Methyl Bromide Treatments for Stored-Product and Quarantine Insects. Annual Review of Entomology, 47, 331-359.

[15] Regnault-Roger, C. (1997). The Potential of Botanical Essential Oils for Insect Pest Control. Integrated Pest Management Reviews, 2, 15-34.

[16] Brower J. H. (1975). Gamma Radiation of Adult Plodia interpunctella: Effects on Mating Sterility and Number of Progeny. Annals of the Entomological Society of America, 68, 1086-1090. 
[17] Brower, J. H. \& Tilton, E. W. (1985). The potential of irradiation as a quarantine treatment for insects infesting stored-food commodities. In Radiation disinfestation of food and agricultural products, 75-86.

[18] Tunçbilek, A. S. (1995). Effect of 60Co Gamma Radiation on the Rice Weevil, Sitophilus oryzae (L.). Anz. Schadlingskd. Pflanzenschutz Umweltschutz, 68, 37-38.

[19] Sharma, M. K. \& Dwivedi S. C. (1997). Investigation on the Effects of Ultraviolet and Infrared Light on the Life Cycle of Callosobruchus chinensis Linn. Journal of Advanced Zoology, 18, 27-31.

[20] Faruki, S. I., Das, D. R. \& Khatun, S. (2005). Effects of UVRadiation on the Larvae of the Lesser Mealworm, Alphitobius diaperinus (Panzer) (Coleoptera: Tenebrionidae) and Their Progeny. Pakistan Journal of Biological Science, 5(4), 444-448.

[21] Ayvaz, A. \& Tunçbilek, A. S. (2006). Effects of Gamma Radiation on Life Stages of the Mediterranean Flour Moth Ephestia kuehniella Zeller (Lepidoptera: Pyralidae). Journal of Pesticide Science, 79, 215-222.

[22] Ayvaz, A., Albayrak, S. \& Tunçbilek, A. S. (2007). Inherited Sterility in Mediterranean Flour Moth Ephestia kuehniella Zeller (Lepidoptera: Pyralidae): Effect of Gamma Radiation Doses on Insect Fecundity Fertility and Developmental Period. Journal of Stored Products Research, 43, 234-239.

[23] Lapidot, M., Saveanu, S., Padova, R. \& Ross, I. (1991). Insect disinfestation by irradiation, in Insect Disinfestation of Food and Agricultural Products by Irradiation. Proceeding IAEA, Vienna, 103.

[24] Ahmed, M. (2001). Disinfestations of Stored Grains Pulses Dried Fruits and Nuts and Other Dried Foods, in Food Irradiation Principles and Applications, ed. by Molins R. John Wiley \& Sons, Inc., New York, NY, 77112.

[25] Halverson S. L., Phillips T. W., Bigelow T. S., Mbata G. N. \& Payton M. E. (1999). The control of various species of stored-product insects with EHF energy. Proceeding of the Annual International Research Conference on Methyl Bromide Alternatives and Emissions Reductions, 541-544.

[26] Azizoğlu, U., Karabörklü, S., Yilmaz, S., Ayvaz, A. \& Temizgül R. (2010). Mikrodalga radyasyonunun Tribolium castaneum Herbst (Coleoptera: Tenebironidae) erginleri üzerindeki öldürücü etkisi. Erciyes Üniversitesi Fen Bilimleri Enstitüsü Dergisi, 26(4), 323-327.

[27] Tilton, E. W. \& Brower, J. H. (1983). Radiation Effects on Arthropods. In: Preservation of Food by Ionizing Radiation. 2, 269-326

[28] Hasan, M. \& Khan, A. R. (1998). Control of stored-product pests by irradiation. Integrated Pest Management Reviews, 3(1), 15-29.

[29] Warchalewski, J. R., Pradzynska, A., Gralik, J. \& Nawrot, J. (2000). The effect of gamma and microwave irradiation of wheat grain on development parameters of some stored grain pests. Nahrung, 44, 411-414.

[30] Çetinkaya, N., Ozyardimci, B., Denli, E. \& Ic, E. (2006). Radiation processing as a post-harvest quarantine control for raisins, dried figs and dried apricots. Radiation Physics and Chemistry, 75, 424-431.

[31] Vadivambal, R., Jayas, D. S. \& White, N. D. G. (2007). Wheat disinfestation using microwave energy. Journal of Stored Products Research, 43, 508-514.

[32] Vadivambal, R., Jayas, D. S. \& White, N. D. G. (2008). Determination of Mortality of Different Life Stages of Tribolium castaneum (Coleoptera: Tenebrionidae) in Stored Barley Using Microwaves. Journal of Economical Entomology, 101(3), 1011-1021.

[33] Valizadegan, O., Pourmirza, A. A. \& Safaralizadeh, M. H. (2009). Combination of microwave radiation and cold storage for control of Oryzalephilus surinamensis (L.) (Coleoptera: Silvanidae). Journal of Biological Sciences, 9, 231-236.

[34] Lu, H., Zhou, J., Xiong, S. \& Zhao, S. (2010). Effects of low-intensity microwave radiation on Tribolium castaneum physiological and biochemical characteristics and survival. Journal of Insect Physiology, 56, 1356-1361.

[35] Bayramzadeh, N. \& Poumirza A. A. (2012). Combined Impacts of Two Type's Cold Storage and Microwave Radiation on Stored Products Insects and Wheat Seed Viability. Egyptian Academic Journal of Biological Science, 5(2), 121-129.

[36] Patil, H., Shejale, K. P., Jabaraj, R., Shah, N. \& Kumar, G. (2020). Disinfestation of red flour beetle (Tribolium castaneum) present in almonds (Prunus dulcis) using microwave heating and evaluation of quality and shelf life of almonds. Journal of Stored Products Research, 87, 101616.

[37] Mills, R. \& Pedersen, J. (1990). A ßour mill sanitation manual. Eagan Press, St. Paul, MN.

[38] Karunakaran, C., Jayas, D. S. \& White, N. D. G. (2004). Identification of wheat kernels damaged by the red flour beetle using X-ray image. Biosystems Engineering, 87(3), 267-274. 
[39] Minitab. (2010). Minitab Inc. MINITAB: Minitab for Windows Version Release 17. State College, PA, USA: Minitab Inc.

[40] Manickavasagan, A., Alahakoon, P. M. K., Al-Busaidi, T. K., Al-Adawi, S., Al-Wahaibi, A. K., Al-Raeesi, A. A., Al-Yahyai, R. \& Jayas, D. S. (2013). Disinfestation of stored dates using microwave energy. Journal of Stored Products Research, 55, 1-5.

[41] Abd El-Raheem, A. M. \& Said, S. M. (2016). Does microwave radiation have an effect on stored product 1nsects and their host food quality?. Academic Journal of Entomology, 9(4), 51-61.

[42] Agha, W. N. A., Amin, A. H., Khidr, S. K. \& Ismail, A. Y. (2017). Entomocidal activity of microwave energy $\&$ some aqueous plant extracts against Tribolium castaneum Herbst \& Trogoderma granarium everts. $6^{\text {th }}$ International Conference and Workshops on Basic and Applied Sciences, AIP Conf. Proc. 1888, 1-13. 